\title{
Atypical Sjögren's Syndrome Initially Presenting as Lymphocytic Interstitial Pneumonitis followed by Immune Thrombocytopenia
}

\author{
Maham Mehmood $\left(D,{ }^{1}\right.$ Abhishrut Jog, ${ }^{1}$ Masooma Niazi, ${ }^{2}$ Arlene Tieng, ${ }^{1,3}$ \\ and Giovanni Franchin ${ }^{1,4}$ \\ ${ }^{1}$ Department of Internal Medicine, BronxCare Health System, Bronx, NY, USA \\ ${ }^{2}$ Department of Pathology, BronxCare Health System, Bronx, NY, USA \\ ${ }^{3}$ Icahn School of Medicine at Mount Sinai, New York, NY, USA \\ ${ }^{4}$ Hofstra University School of Medicine, Long Island, NY, USA \\ Correspondence should be addressed to Maham Mehmood; mahum.arshad89@gmail.com
}

Received 18 November 2020; Accepted 6 March 2021; Published 15 March 2021

Academic Editor: Mehmet Soy

Copyright (C) 2021 Maham Mehmood et al. This is an open access article distributed under the Creative Commons Attribution License, which permits unrestricted use, distribution, and reproduction in any medium, provided the original work is properly cited.

\begin{abstract}
Background. Sjögren's syndrome is an autoimmune disease characterized primarily by decreased exocrine gland function leading to eye and mouth dryness. Extraglandular manifestations occur less frequently. Case Report. A 74-year-old man with hypertension was admitted with productive cough and fever. On physical examination, he had bilateral lower lung decreased breath sounds. A chest radiograph showed bibasilar patchy infiltrate. Laboratory studies revealed hemoglobin of $11.9 \mathrm{~g} / \mathrm{dL}$, white blood cell count of 16,000/uL, and platelet count of 250,000/uL. Empiric antibiotic therapy was begun for suspected community acquired pneumonia, and then he was discharged home. However, his cough recurred. Chest computed tomography demonstrated adenopathy throughout the mediastinum and multiple ill-defined patchy groundglass opacities with a lower lobe prominence. He underwent a transbronchial biopsy to rule out malignancy; however, it showed lymphocytic interstitial pneumonitis. Antinuclear antibody was 1:80 homogeneous, and anti-SSA antibody was 6.3 AI (normal $<1.0 \mathrm{AI}$ ). The patient was treated with prednisone $20 \mathrm{mg}$ /day with marked improvement in his symptoms. Repeat chest computed tomography showed decreased groundglass opacities and decreased mediastinal lymph nodes. After more than a year, he was readmitted due to petechiae on his buccal mucosa and a platelet count of $2000 / \mu \mathrm{L}$. The patient was started on prednisone $80 \mathrm{mg} / \mathrm{d}$ and intravenous immunoglobulin $80 \mathrm{~g} / \mathrm{d}$ for 2 consecutive days. The platelet count eventually increased to $244,000 / \mu \mathrm{L}$. Conclusion. We report a rare presentation of Sjogren's syndrome manifesting as acute lymphocytic interstitial pneumonitis and followed by immune thrombocytopenia. Both extraglandular manifestations responded well to corticosteroid therapy.
\end{abstract}

\section{Background}

Sjögren's syndrome is a chronic systemic autoimmune disorder commonly characterized by dry eyes and dry mouth. It has an incidence rate of 7/100,000 persons annually [1]. In addition to the anti-SSA antibody, the oral and ocular symptoms are assessed in the most recent classification criteria for primary Sjögren's syndrome [2]. However, extraglandular features may occur in a third of the cases and sometimes they are the presenting signs. These can include Raynaud phenomenon or cutaneous vasculitis, arthralgia or myopathy, interstitial lung disease (ILD), primary biliary cholangitis, interstitial nephritis, peripheral neuropathy, or lymphoma [3]. ILD is seen in only 9-20\% cases of Sjögren's syndrome, and of the types of ILD, lymphocytic interstitial pneumonitis (LIP) is less frequently identified [4]. LIP was first described in 1966 [5]. As opposed to pulmonary manifestations in Sjögren's syndrome, immune thrombocytopenia (ITP) is not as well-recognized a feature. To the best of our knowledge, we know of no case report of LIP and ITP in Sjögren's syndrome. We present one such case of Sjögren's syndrome that presented as LIP and then developed ITP. 


\section{Case Report}

A 74-year-old Ecuadorian man who smoked 3 cigarettes a day for a year but quit 15 years prior presented to the Emergency Department (ED) with four days of a productive cough that did not improve with azithromycin. His medical history included hypertension and cataracts. He worked at a clothing factory for years. The body mass index was $24.9 \mathrm{~kg} /$ $\mathrm{m}^{2}$. Physical examination showed a blood pressure of 154/ $86 \mathrm{mmHg}$, pulse of 115 beats/minute, respiration rate of 18/ min, oxygen saturation of $95 \%$ on ambient air, and temperature of $101^{\circ} \mathrm{F}$. Pulmonary auscultation on hospital admission revealed decreased bilateral breath sounds in the lower lung fields. A chest radiograph showed a large rounded opacity in the superior segment right lower lobe and bibasilar patchy infiltrate. The results of laboratory studies revealed a white blood cell count of $16,000 / \mu$ l with $82.7 \%$ neutrophils and $8 \%$ lymphocytes, hemoglobin of $11.9 \mathrm{~g} / \mathrm{dl}$, and platelet count of $250,000 / \mu \mathrm{l}$. Blood and urine cultures yielded no growth, and his acute symptoms of fever and cough subsided with ceftriaxone plus azithromycin. He was discharged home three days after admission. However, his productive cough recurred. More than a month later, computed tomography (CT) scan of the chest revealed multiple, approximately $16.4-28.6 \mathrm{~mm}$, enlarged nodes in the mediastinum and hila, diffuse septal thickening, and multiple ill-defined patchy ground glass opacities with a lower lobe predominance (Figure 1). On transthoracic echocardiography, the systolic pressure of the pulmonary artery was $33 \mathrm{mmHg}$ with normal ejection fraction. The rheumatoid factor was negative, and the antinuclear antibody was positive at $1: 80$ with a homogeneous pattern. The patient was readmitted ( 2 months after the first episode) due to pleuritic chest pain and dyspnea on exertion. Radiography of the chest revealed bilateral prominent lung interstitial markings particularly in the left perihilar areas, and he was empirically covered for hospital-acquired pneumonia with vancomycin and piperacillin/tazobactam. More than a month later, his C-reactive protein (CRP) was $<5 \mathrm{mg} / \mathrm{l}$. He was lost to follow-up for five months but returned complaining of xerophthalmia, and artificial tears provided relief. He was again lost to follow-up for another five months and then noted to have weight loss of 25 pounds over 6 months. Follow-up chest CT findings more than a year after his initial admission included worsening septal thickening and ground glass opacities. Transbronchial lung biopsy demonstrated mild thickening of interstitial septae and increased infiltration by lymphocytes, macrophages, and plasma cells (Figure 2) consistent with LIP. No malignant cells were seen.

Then, he developed bilateral metacarpophalangeal swelling and pain and swelling. To treat LIP, he was started on oral prednisone at a dose of $20 \mathrm{mg} /$ day $(0.25 \mathrm{mg} / \mathrm{kg})$. His joint symptoms improved markedly. A laboratory workup was positive for the SSA antibody, and he was diagnosed with Sjögren's syndrome. His CRP was elevated $(191 \mathrm{mg} / \mathrm{l})$ and complement levels were low (C3 and C4, $40 \mathrm{mg} / \mathrm{dl}$ and $1 \mathrm{mg} / \mathrm{dl}$, respectively). He tested negative for anti-SSB, anti-Jo-1, anti Scl-70, anti-neutrophil cytoplasmic, and

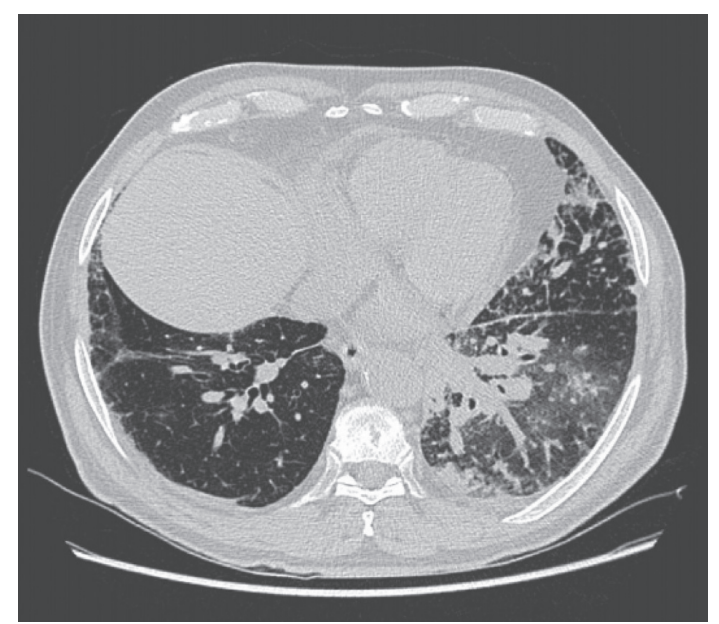

FIGURE 1: Computed tomography of chest showing transverse view of lungs with multiple patchy ground glass opacities with predominant lower lobe involvement, more than a month after initial presentation.

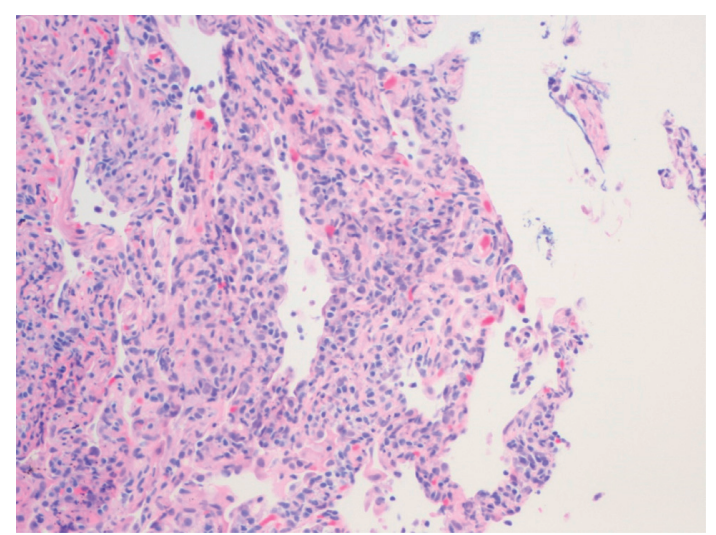

Figure 2: Pathological findings of lymphocytic interstitial pneumonitis. High-power analysis of lung tissue shows interstitial septa thickening, infiltration by small- to medium-sized lymphocytes (hematoxylin-eosin, magnification $\times 200$ ).

anti-double-stranded DNA antibodies. Pulmonary function testing did not show obstructive dysfunction. His postbronchodilator forced expiratory volume in one second $\left(\mathrm{FEV}_{1}\right)$ was 3 liters (126\% predicted), total lung capacity (TLC) was 5.48 liters (104\% predicted), and diffusion capacity for carbon monoxide (DLCO) was $17.8 \mathrm{ml} / \mathrm{min} /$ $\mathrm{mmHg}$ ( $82 \%$ predicted). The patient complained of dry mouth, which improved with pilocarpine. He was lost to follow-up for four months, during which time he discontinued prednisone after five months. Then, he developed anterior uveitis which was treated successfully with topical prednisolone acetate. A follow-up chest CT scan more than a year after oral prednisone was first started showed bibasilar and lingular interstitial fibrotic changes, decreased prominence of associated interlobular septal thickening and ground glass opacity, and a decrease in the size of the lymph nodes. Prednisone $20 \mathrm{mg} /$ day was resumed and slowly tapered over three months. A month after prednisone was tapered to $10 \mathrm{mg}$, he presented to the ED with oral mucosal 
bleeding. His vital signs were unremarkable. Physical examination was significant for a bloody oral cavity, bruises on the right arm and legs, and scattered petechiae on the chest and lower legs. Laboratory tests were remarkable for a platelet count of 2000/ $\mu$ l. Peripheral smear did not show schistocytes. CT head was negative for acute hemorrhage. Further labs, including liver function, lactate dehydrogenase, and vitamin B12 levels, were normal. Hepatitis C antibody, hepatitis B surface antigen, and human immunodeficiency virus were negative. No obvious drug-related causes of thrombocytopenia were identified. Blood and urine cultures were negative. A diagnosis of ITP secondary to Sjögren's syndrome was made. A bone marrow biopsy was not done.

The patient was started on prednisone at $80 \mathrm{mg} /$ day $(1 \mathrm{mg} /$ $\mathrm{kg} / \mathrm{d}$ ) and received a total of 12 units of platelet transfusion. He was given intravenous immunoglobulin (IVIG) at $1 \mathrm{~g} / \mathrm{kg} / \mathrm{d}$ for two days. His platelet count improved to $84,000 / \mu \mathrm{l}$ by the sixth day, and oral bleeding resolved. The patient was discharged home, but he was unable to continue the prednisone because the prescription was accidentally not transmitted to the pharmacy. After five days, he was readmitted with oral mucosal bleeding. The platelet count was found to be $3000 / \mu \mathrm{l}$. He received IVIG at $1 \mathrm{~g} / \mathrm{kg} / \mathrm{d}$ and IV methylprednisolone $62.5 \mathrm{mg}$ twice a day. By the fifth day, his platelet count was $178,000 / \mu \mathrm{l}$. He was discharged on prednisone $80 \mathrm{mg} /$ day, and it was uneventfully tapered off over four months. He was also started on hydroxychloroquine for musculoskeletal pain. His latest platelet count around six months after the last admission was 254,000 cells/ $\mu 1$.

\section{Discussion}

We present the case of a 74-year-old man with Sjögren's syndrome who developed LIP, arthritis, uveitis, and ITP in addition to the sicca symptoms. The initial presumptive diagnosis of community acquired pneumonia led to further workup after his initial chest X-ray was suspicious for malignancy. A chest CT scan unexpectedly showed multiple enlarged nodes in the mediastinum and hila as well as ground glass opacities in a man who did not have an extensive smoking history. Given his significant weight loss, a transbronchial lung biopsy was performed to evaluate for malignancy or any other underlying pathology. In this case, LIP was diagnosed and that warranted a thorough rheumatologic investigation. Sjögren's syndrome was diagnosed after malignancy was ruled out. Hypocomplementemia can be associated with systemic disease activity, and indeed this patient had involvement of the lungs, joints, eye, and platelets. LIP is characterized by the proliferation of lymphocytes and plasma cells in lung interstitium with lymphoid follicles and germinal centers. Most patients present with dyspnea, cough, and inspiratory crackles on lung exam. LIP is considered benign but can progress to lymphoma or end-stage lung disease. Prednisone can be administered for at least six months, and rituximab has been found to effective [6].

The autoimmune disease more well known to be associated with thrombocytopenia is systemic lupus erythematosus (SLE), so much so that it is one of the elements in its classification criteria [7]. Thrombocytopenia in Sjögren's syndrome is not as well recognized, and it is exceedingly rare to find severe thrombocytopenia (platelet count $<50,000$ cells $/ \mu \mathrm{l}$ ) in Sjögren's syndrome. In a retrospective study by Ramos-Casals et al. of 380 patients with Sjögren's syndrome, severe thrombocytopenia was found in only $0.4 \%$ [8].

In 1950 in St. Louis, the hematology fellow William Harrington injected himself with the plasma from a patient with ITP [9]. This led to a drastic drop in his platelets, which supported a role for autoantibodies in the pathophysiology of the disease. ITP in Sjögren's syndrome can be explained by peripheral destruction of platelets by the circulating antibodies. Sjögren's syndrome and SLE, both with circulating antibodies, are more prone to cause such destruction.

Glucocorticoids are the cornerstone of the management of ITP. Of note, this patient developed ITP after the prednisone for his LIP was tapered. Our patient showed a remarkable response with steroids and IVIG. In refractory cases of Sjögren's syndrome with ITP, rituximab or splenectomy can be considered. Our patient suffered from two major extraglandular manifestations that occurred serially, not simultaneously. The administration of a steroid-sparing agent such as rituximab can be considered a potential treatment for his pulmonary and hematologic involvement.

\section{Conclusion}

The classic features of Sjögren's syndrome include xerophthalmia and xerostomia, but they may not always lead to the diagnosis as they may present later in the course of the illness. The initial absence of these features should not deter testing for Sjögren's syndrome, especially if there is suspicious evidence for ILD. Furthermore, Sjögren's syndrome is a rare cause of ITP. LIP and ITP can respond well to steroids. In Sjögren's syndrome, rituximab can be considered with one serious extraglandular organ involvement, in hopes of preventing additional systems to be severely affected.

\section{Disclosure}

This abstract was presented at the American College of Allergy, Asthma, \& Immunology (ACAAI) Annual Scientific Meeting on November 7-11, 2019, in Houston, Texas, USA.

\section{Conflicts of Interest}

The authors declare that they have no conflicts of interest.

\section{References}

[1] B. Qin, J. Wang, Z. Yang et al., "Epidemiology of primary Sjögren's syndrome: a systematic review and meta-analysis," Annals of the Rheumatic Diseases, vol. 74, no. 11, pp. 1983-1989, 2015.

[2] C. H. Shiboski, S. C. Shiboski, R. Seror et al., "2016 American College of Rheumatology/European League against rheumatism classification criteria for primary Sjögren's syndrome: a consensus and data-driven methodology involving three 
international patient cohorts," Arthritis \& Rheumatology, vol. 69, no. 1, pp. 35-45, 2017.

[3] J. Jameson, A. Fauci, D. Kasper, S. Hauser, D. Longo, and J. Loscalzo, Harrison's Principles of Internal Medicine, McGraw-Hill Education/Medical, New York, NY, USA, 12th edition, 2018.

[4] M. Ramos-Casals, P. Brito-Zerón, R. Seror et al., "Characterization of systemic disease in primary Sjögren's syndrome: EULAR-SS Task Force recommendations for articular, cutaneous, pulmonary and renal involvements [published correction appears in Rheumatology (Oxford)," Rheumatology, vol. 56, no. 7, p. 1245, 2017.

[5] C. B. Carrington and A. A. Liebow, "Lymphocytic interstitial pneumonia," The American Journal of Pathology, vol. 48, p. 36a, 1966.

[6] R. Seror, C. Sordet, L. Guillevin et al., "Tolerance and efficacy of rituximab and changes in serum B cell biomarkers in patients with systemic complications of primary Sjogren's syndrome," Annals of the Rheumatic Diseases, vol. 66, no. 3, pp. 351-357, 2007.

[7] M. Aringer, K. Costenbader, D. Daikh et al., "2019 European League against Rheumatism/American College of Rheumatology classification criteria for systemic lupus erythematosus," Annals of the Rheumatic Diseases, vol. 78, no. 9, pp. 1151-1159, 2019.

[8] M. Ramos-Casals, J. Font, M. García-carrasco et al., "Primary Sjögren syndrome,” Medicine, vol. 81, no. 4, pp. 281-292, 2002.

[9] D. S. Beardsley, "Pathophysiology of immune thrombocytopenic purpura," Blood Reviews, vol. 16, no. 1, pp. 13-14, 2002. 